\title{
Functional background of the Tsallis entropy: "coarse-grained" systems and "kappa" distribution functions
}

\author{
A. V. Milovanov and L. M. Zelenyi \\ Space Research Institute, 117810 Moscow, Russia
}

Received: 17 November 1999 - Revised: 28 February 2000 - Accepted: 22 March 2000

\begin{abstract}
The concept of the generalized entropy is analyzed, with the particular attention to the definition postulated by Tsallis [J. Stat. Phys. 52, 479 (1988)]. We show that the Tsallis entropy can be rigorously obtained as the solution of a nonlinear functional equation; this equation represents the entropy of a complex system via the partial entropies of the subsystems involved, and includes two principal parts. The first part is linear (additive) and leads to the conventional, Boltzmann, definition of entropy as the logarithm of the statistical weight of the system. The second part is multiplicative and contains all sorts of multilinear products of the partial entropies; inclusion of the multiplicative terms is shown to reproduce the generalized entropy exactly in the Tsallis sense. We speculate that the physical background for considering the multiplicative terms is the role of the long-range correlations supporting the "macroscopic" ordering phenomena (e.g., formation of the "coarse-grained" correlated patterns). We prove that the canonical distribution corresponding to the Tsallis definition of entropy, coincides with the so-called "kappa" distribution which appears in many physical realizations. This has led us to associate the origin of the "kappa" distributions with the "macroscopic" ordering ("coarse-graining") of the system. Our results indicate that an application of the formalism based on the T'sallis notion of entropy might actually have sense only for the systems whose statistical weights, $\Omega$, are relatively small. (For the "coarse-grained" systems, the weight $\Omega$ could be interpreted as the number of the "grains".) For large $\Omega$ (i.e., $\Omega \rightarrow \infty$ ), the standard statistical mechanical formalism is advocated, which implies the conventional, Boltzmann definition of entropy as $\ln \Omega$.
\end{abstract}

Correspondence to: A. V. Milovanov

\section{Introduction}

The concept of entropy plays a fundamental role in statistical mechanics and information theory. The standard definition of the entropy $S$ for a discrete probability distribution reads [see, e.g., Landau and Lifshitz (1970)]

$S\left[\left\{p_{i}\right\}\right]=-\sum_{i=1}^{\Omega} p_{i} \ln p_{i}$

where $\Omega$ is the total number of the possible (microscopic) states, and $\left\{p_{i}\right\}$ are the associated probabilities obeying $\sum_{i=1}^{\Omega} p_{i}=1$. Entropy $S\left[\left\{p_{i}\right\}\right]$ is extremized in the case of equiprobability, i.e., $p_{i}=1 / \Omega$ for all $i=1, \ldots, \Omega$; this immediately recovers the Boltzmann expression

$S^{B}=\ln \Omega$.

Considerable advances in the theory of dynamical systems [Sinai (1972)] that have lead to a beginning of a dynamical formulation of statistical mechanical problems [Ruelle (1978), Eckmann and Ruelle (1985), Gaspard and Dorfman (1995)], especially for nonequilibrium systems, near or far from equilibrium, opened new perspectives of the thermodynamic concepts [Paladin and Vulpiani (1987), McCauley (1990), Badii and Politi (1997)]. In this context, the importance of a generalized entropy [Badii and Politi (1997), Schroeder (1991)] was recognized, particularly in describing the mechanisms for the transition to chaos [Paladin and Vulpiani (1987)].

A suitable realization for a generalized entropy was found by the Hungarian mathematician Renyi [see, e.g., Renyi (1955), Renyi (1970)] who introduced the following expression based on the moments of order $q$ of the probabilities $p_{i}$ [cf. Eq. (1)]:

$S_{q}^{R}\left[\left\{p_{i}\right\}\right]=-\frac{1}{q-1} \ln \sum_{i=1}^{\Omega} p_{i}^{q}$. 
Here, $q$ is a real number; for $q \rightarrow 1$, definition (3) recovers the standard expression (1). The Renyi entropy $S_{q}^{R}\left[\left\{p_{i}\right\}\right]$ is a practical tool for analyzing temporal intermittency in chaotic systems [Paladin and Vulpiani (1987)], i.e., the type of motion when a regular behavior in phase space for long times is interrupted by randomly distributed bursts of strong chaoticity. In the case of equiprobability $\left(p_{i}=1 / \Omega\right.$ for all $\left.i=1, \ldots, \Omega\right)$, the entropy $S_{q}^{R}\left[\left\{p_{i}\right\}\right]$ is reduced to the Boltzmann form $S^{B}=\ln \Omega$ independently of $q$.

An intriguing generalization of entropy (1) was proposed by Daroczy (1970) and later revived by Tsallis (1988). Tsallis (1988) postulated for the entropy

$S_{q}^{T}\left[\left\{p_{i}\right\}\right]=\frac{1}{q-1}\left[1-\sum_{i=1}^{\Omega} p_{i}^{q}\right]$

where $q$ is a real number, and $\sum_{i=1}^{\Omega} p_{i}=1$.

It is straightforward to verify [Tsallis (1988)] that $S_{q}^{T}\left[\left\{p_{i}\right\}\right]$ is extremized, for all values of $q$, in the case of equal probability distribution, i.e., $p_{i}=1 / \Omega$ for all $i=1, \ldots, \Omega$, yielding

$S_{q}^{T}=\frac{\Omega^{1-q}-1}{1-q}$.

Expression (5) is reduced to the Boltzmann expression $S^{B}=\ln \Omega$ only in the limit $q \rightarrow 1$. The entropy $S_{q}^{T}$ given by Eq. (5) diverges if $q \leq 1$ and saturates at $1 /(q-1)$ if $q>1$, in the limiting case of $\Omega \rightarrow \infty$.

Much theoretical effort has been made in the recent years to prove ar disprove the physical relevance of the Tsallis definition of entropy (4),(5). On one hand, the properly generalized formulation of the Ehrenfest theorem and Jaynes duality relations [Plastino and Plastino (1993)], fluctuation-dissipation theorem [da Silva et al. (1993), Rajagopal (1996)], and Bogolyubov inequality [Plastino and Tsallis (1993)] has been proposed. The validity of the $H$-theorem for the Tsallis entropy (4),(5) was analyzed by Mariz (1992) and Ramshaw (1993ab). The generalized microcanonical and canonical distributions were discussed in the original paper of Tsallis (1988), and for quantum statistics, by Buyukkilic and Demirhan (1993). In the meantirne, the Tsallis definition (4),(5) still lacks the proper probabilistic substantiation and observes certain characteristics that may cause a concern; amongst them is the allowed violation of the second law [Treumann (1999a), Treumann (1999b)]. Direct applications of the Tsallis entropy have been, nevertheless, found in fractal random walks [Alemany and Zanette (1993), Zanette and Alemany (1995)], hydrodynamic turbulence [Boghosian (1996)], cosmic background radiation [Hamity and Barraco (1996)], and others. The validity of the Tsallis notion of entropy $(4),(5)$ thus remains a delicate open question in the modern statistical physics, and its detailed comprehension requires further research.
A remarkable property of the Tsallis entropy (4),(5) is its nonadditivity [Tsallis (1988)]. This suggests a treatment of the Tsallis entropy (4),(5) as a proper instrument in dealing with a class of physical systems which exhibit long-range correlations. The long-range correlations could be associated with the extensive interactions in the system; relevant examples might be found, for instance, in self-organized criticality [Chang (1999)], "fractional" resonances [Milovanov and Zelenyi (1998)], coherent reconnection [Buchner (1998)], developed turbulence [Zelenyi et al. (1998), Milovanov et al. $(1996,2000)$ ], gravitation [Saslaw (1987), Spitzer (1987)], anion dynamics [Haldane (1991)], percolation [Isichenko (1992), Nakayama et al. (1994)], etc.

The concept of anions, the particles having "fractional" statistics, is the indirect prediction of the topological three-dimensional gauge theories studied by Witten (1989); the development of the quantum statistics of anions has stimulated enormous progress in the condensed matter physics (e.g., the quantum Hall effect and high-temperature superconductivity).

Topological ideas have been recently applied by Milovanov (1997) to an analysis of the structures characterized by a diverging correlation length, e.g., percolating sets at the threshold of percolation; the sinthesis of the standard percolation theory and the homotopic/differential topology of manifolds leads to the important conclusions regarding the geometric properties of the intrinsically correlated fields [Milovanov and Zimbardo (2000)].

In this paper, we show that the property of nonadditivity is a key to an understanding of the statistical background of the Tsallis definition of entropy (4),(5). In section 2, we formalize this property in terms of a nonlinear functional equation whose solution is proven to be the Tsallis entropy $S_{q}^{T}$ where the parameter $q$ (the so-called "Tsallis index" [Alemany and Zanette (1993)]) defines the "degree of nonlinearity" of this equation. In section 3 , we establish the canonical distribution for a statistical system whose entropy is given by $S_{q}^{T}$; we demonstrate that this distribution coincides with the socalled "kappa" distribution which attracts a good deal of attention in literature. We summarize the results obtained in section 4 of the paper.

\section{Tsallis Entropy from Functional Equation}

Consider two statistical systems $\mathcal{M}^{\prime}$ and $\mathcal{M}^{\prime \prime}$ whose statistical weights (i.e., the total numbers of the possible microscopic states for each system) are, respectively, $\Omega^{\prime}$ and $\Omega^{\prime \prime}$. Let $S^{\prime}\left(\Omega^{\prime}\right)$ and $S^{\prime \prime}\left(\Omega^{\prime \prime}\right)$ denote the entropies of the systems $\mathcal{M}^{\prime}$ and $\mathcal{M}^{\prime \prime}$. We assume below that the microscopic configurations for both $\mathcal{M}^{\prime}$ and $\mathcal{M}^{\prime \prime}$ are equiprobable; this means that all the microscopic probabilities $p_{i}^{\prime}$ for the system $\mathcal{M}^{\prime}$ are equal to $1 / \Omega^{\prime}$, and all the microscopic probabilities $p_{i}^{\prime \prime}$ for the system $\mathcal{M}^{\prime \prime}$, to 
$1 / \Omega^{\prime \prime}$.

Assuming statistical independence of the systems $\mathcal{M}^{\prime}$ and $\mathcal{M}^{\prime \prime}$, we may define the entropy of the complex system $\mathcal{M}=\mathcal{M}^{\prime} \cup \mathcal{M}^{\prime \prime}$ as an additive function of the entropies of its constituents, $\mathcal{M}^{\prime}$ and $\mathcal{M}^{\prime \prime}$. Thus, we may write

$S(\Omega)=S^{\prime}\left(\Omega^{\prime}\right)+S^{\prime \prime}\left(\Omega^{\prime \prime}\right)$

where $S(\Omega)$ is the entropy of the entire system $\mathcal{M}=$ $\mathcal{M}^{\prime} \cup \mathcal{M}^{\prime \prime}$, and $\Omega=\Omega^{\prime} \Omega^{\prime \prime}$ is the statistical weight of $\mathcal{M}$. It is well known from the standard courses of statistical mechanics [see, e.g., Levich (1962), Reif (1965)] that the functional equation (6) immediately leads to the Boltzmann definition of entropy for the system $\mathcal{M}$, i.e., $S(\Omega) \equiv S^{B}=\ln \Omega$ [see Eq. (2)].

It is clear that an inclusion of the long-range correlations that might be present in the system $\mathcal{M}$ in general, violates the linear expansion (6). Indeed, such correlations appear in an essential mutual influence of the systems $\mathcal{M}^{\prime}$ and $\mathcal{M}^{\prime \prime}$ which perturbs the microscopic states throughout $\mathcal{M}$. Hence, the systems $\mathcal{M}^{\prime}$ and $\mathcal{M}^{\prime \prime}$ cannot be treated as statistically independent when the longrange correlations play a role; the entropy of the complex system $\mathcal{M}=\mathcal{M}^{\prime} \cup \mathcal{M}^{\prime \prime}$ cannot be then reduced to the algebraic sum (6) of the entropies of the constituent systems $\mathcal{M}^{\prime}$ and $\mathcal{M}^{\prime \prime}$. Note that the relation $\Omega=\Omega^{\prime} \Omega^{\prime \prime}$ may be also violated in this case unless additional restrictions are implied (see the consideration below).

Taking account of the correlations in the system $\mathcal{M}$ would mean that expansion (6) of the entropy $S(\Omega)$ must be supplemented by the multiplicative terms, each hav: ing the form $\omega(\lambda, \mu)\left[S^{\prime}\left(\Omega^{\prime}\right)\right]^{\lambda}\left[S^{\prime \prime}\left(\Omega^{\prime \prime}\right)\right]^{\mu}$ where $\lambda$ and $\mu$ are some nonzero constants depending on the particular nature of the system $\mathcal{M}$, and $\omega(\lambda, \mu)$ is some function of $\lambda$ and $\mu$. Below, we are restricting ourselves to the "natural" case of $\lambda=\mu$, when the correlations are symmetric over $\mathcal{M}^{\prime}$ and $\mathcal{M}^{\prime \prime}$. Each of the additional multiplicative terms could be then represented as $\omega(\lambda, \lambda)\left[S^{\prime}\left(\Omega^{\prime}\right) S^{\prime \prime}\left(\Omega^{\prime \prime}\right)\right]^{\lambda}$.

Our further interest is concentrated on the relatively weak correlations when the multiplicative terms are linear over each of the partial entropies, $S^{\prime}\left(\Omega^{\prime}\right)$ and $S^{\prime \prime}\left(\Omega^{\prime \prime}\right)$. In this case, the only multiplicative term to be kept in the expansion of the entropy $S(\Omega)$ must be the quadratic one, i.e., $\omega(1,1) S^{\prime}\left(\Omega^{\prime}\right) S^{\prime \prime}\left(\Omega^{\prime \prime}\right)$, for which the power exponent $\lambda$ is equal to unity $(\lambda=1)$. [The multiplicative term $\omega(1,1) S^{\prime}\left(\Omega^{\prime}\right) S^{\prime \prime}\left(\Omega^{\prime \prime}\right)$ is thus given by the bilinear function of the partial entropies $S^{\prime}\left(\Omega^{\prime}\right)$ and $S^{\prime \prime}\left(\Omega^{\prime \prime}\right)$.] Consequently, we have, instead of Eq. (6),

$S(\Omega)=S^{\prime}\left(\Omega^{\prime}\right)+S^{\prime \prime}\left(\Omega^{\prime \prime}\right)+\omega S^{\prime}\left(\Omega^{\prime}\right) S^{\prime \prime}\left(\Omega^{\prime \prime}\right)$

where $\omega \equiv \omega(1,1)$ is a constant to be quantified below.

Eq. (7) can be easily generalized to give an expansion of the entropy $S(\Omega)$ for a complex system $\mathcal{M}=$ $\bigcup_{i=1}^{N} \mathcal{M}^{\prime \ldots[i] \ldots \prime} \equiv \mathcal{M}^{\prime} \cup \mathcal{M}^{\prime \prime} \cup \ldots \cup \mathcal{M}^{\prime \ldots[N] \ldots \prime}$ composed of an arbitrary number $N \geq 2$ of the mutually correlated systems $\mathcal{M}^{\prime}, \mathcal{M}^{\prime \prime}, \ldots, \overline{\mathcal{M}}^{\prime \ldots[N] \ldots \prime}$. (The superscript "f...[i]..." means that the prime " $l$ " is repeated $i$ times.) In fact, denote $S^{\prime \ldots[i] \ldots t} \equiv S^{\prime \ldots[i] \ldots \prime}\left(\Omega^{\prime \ldots[i] \ldots t}\right)$ to be the entropy of the system $\mathcal{M}^{\prime \ldots[i] \ldots t}$ where the index $i$ runs from 1 to $N$, and $\Omega^{\prime \ldots[i] \ldots t}$ be the statistical weight of $\mathcal{M}^{\prime \ldots[i] \ldots \prime}$. Assuming the cases of equiprobability for each $\mathcal{M}^{\prime \ldots[i] \ldots \prime}$ and making use of Eq. (7), after $N-2$ iterations one gets the functional equation

$$
\begin{aligned}
S(\Omega) & =\sum_{i} S^{\prime \ldots[i] \ldots \prime}+\omega \sum_{i<j} S^{\prime \ldots[i] \ldots \prime} S^{\prime \ldots[j] \ldots \prime}+ \\
& +\omega^{2} \sum_{i<j<k} S^{\prime \ldots[i] \ldots \prime} S^{\prime \ldots[j] \ldots \prime} S^{\prime \ldots[k] \ldots \prime}+\cdots+ \\
& +\omega^{N-1} S^{\prime} S^{\prime \prime} \times \ldots \times S^{\prime \ldots[N] \ldots \prime}
\end{aligned}
$$

where all the indices $i, j, k, \ldots=1, \ldots, N$, and the arguments, $\Omega^{\prime \ldots[i] \ldots \prime}$, of all $S^{\prime \ldots[i] \ldots t}$ are omitted for simplicity. Eq. (8) takes into account the long-range correlations of all the orders from 2 to $N \geq 2$ effective in the complex system $\mathcal{M}=\bigcup_{i=1}^{N} \mathcal{M}^{\prime \ldots[i] \ldots}$. For instance, the pair correlations are contained in $C_{N}^{2}=N ! / 2 !(N-2)$ ! bilinear terms of the form $\omega S^{\prime \ldots[i] \ldots \prime} S^{\prime \ldots[j] \ldots t}, i<j$; the triple correlations, in $C_{N}^{3}=N ! / 3 !(N-3)$ ! trilinear terms of the form $\omega^{2} S^{\prime \ldots[i] \ldots \prime} S^{\prime \ldots[j] \ldots \prime} S^{\prime \ldots[k] \ldots 1}, i<j<k ; \ldots$; the correlations of the highest order $N$, in $C_{N}^{N}=1 N$-linear term $\omega^{N-1} S^{\prime} S^{\prime \prime} \times \ldots \times S^{\prime \ldots[N] \ldots \prime}$. (Here, the quantities $C_{N}^{n}, n=2,3, \ldots, N$, denote the binomial coefficients.)

The correlations of arbitrary order $2 \leq n \leq N$ would be contained in $C_{N}^{n}=N ! / n !(N-n) ! n$-linear products, $\omega^{n-1} S^{\prime \ldots[i] \ldots \prime} S^{\prime \ldots[j] \ldots \prime} \times \ldots \times S^{\prime \ldots[l] \ldots \prime}, i<j<\ldots<l$, of $n$ partial entropies $S^{\prime \ldots[i] \ldots t}, S^{\ldots[j] \ldots t} \ldots, S^{\prime \ldots[l] \ldots l}$ for $n$ different systems $\mathcal{M}^{\prime \ldots[i] \ldots t}, \mathcal{M}^{\prime \ldots[j] \ldots t}, \ldots, \mathcal{M}^{\prime \ldots[l] \ldots t}$. A suitable summation of the terms $\omega^{n-1} S^{\prime \ldots[i] \ldots} S^{\prime \ldots[j] \ldots \prime} \times$ $\ldots \times S^{\prime \ldots[l] \ldots \prime}$ for each $2 \leq n \leq N$ could be done over the ranked permutations of the corresponding indices $i, j, \ldots$, $l$, e.g., over the increasing sequences $i<j<\ldots<l$ of the indices $i, j, \ldots, l$ (see above), just to avoid inclusion of the identical terms into Eq. (8). The total number of the multiplicative terms of all the orders $n$ from 2 to $N$ on the right of Eq. (8) is easily seen to be $\sum_{n=2}^{N} C_{N}^{n}=$ $2^{N}-(N+1)$.

It is important to note that $\mathrm{Eq} . \quad(8)$ is linear over each of the partial entropies $S^{\prime \ldots[i] \ldots,}, i=1, \ldots, N$, since it contains only multilinear products of $S^{\prime \ldots[i] \ldots l}$ for any $2 \leq n \leq N$. This means the following: The correlations involved, although effective up to the highest order $N \geq 2$, are not strong enough to change the statistical weights $\Omega^{\prime \ldots[i] \ldots t}$ of the systems $\mathcal{M}^{\prime \ldots[i] \ldots t}$. In other words, Eq. (8) implies that all $\mathcal{M}^{\prime \ldots[i] \ldots \prime}, i=1, \ldots, N$, have the same statistical weights $\Omega^{\prime \ldots[i] \ldots \prime}$ as they would do in absence of the correlations, i.e., when all $\mathcal{M}^{\prime \ldots[i] \ldots t}$ are isolated from each other. The correlations in the complex system $\mathcal{M}=\bigcup_{i=1}^{N} \mathcal{M}^{\prime \ldots[i] \ldots \prime}$ could then only alter the probabilities of the inherent microscopic configurations of the systems $\mathcal{M}^{\prime \ldots[i] \ldots \prime}$, rather than affect the statistical weights $\Omega^{\prime \ldots[i] \ldots t}$ themselves. A description 
of the sufficiently strong long-range correlation mechanisms that could change the statistical weights $\Omega^{\prime \ldots[i] \ldots \prime}$ for the systems $\mathcal{M}^{\prime \ldots[i] \ldots \prime}, i=1, \ldots, N$, i.e., result in a substantial nonlinear reorganization of the allowed microscopic configurations for all $\mathcal{M}^{\prime \ldots[i] \ldots \prime}$, would require an analysis of more general functional equations, with the partial entropies $S^{\prime \ldots[i] \ldots t}$ nonlinearly present in the corresponding expansions of $S(\Omega)$.

Since expansion (8) of the entropy $S(\Omega)$ is the linear function over each of the entropies $S^{\prime \ldots[i] \ldots t}\left(\Omega^{\prime \ldots[i] \ldots t}\right)$, $i=1, \ldots, N$, we can preserve below the standard relation between the statistical weight $\Omega$ of the complex system $\mathcal{M}=\bigcup_{i=1}^{N} \mathcal{M}^{\prime \ldots[i] \ldots \prime}$ and the partial weights $\Omega^{\prime \ldots[i] \ldots \prime}$. This is given by the $N$-linear product [Landau and Lifshitz (1970)]

$\Omega=\prod_{i=1}^{N} \Omega^{\prime \cdots[i] \ldots \prime}$

This expression should be substituted into Eq. (8). It is widely known that relation (9) holds for the complex system $\mathcal{M}=\bigcup_{i=1}^{N} \mathcal{M}^{\prime \ldots[i] \ldots t}$ composed of the statistically independent $\mathcal{M}^{\prime \ldots[i] \ldots \prime}, i=1, \ldots, N$. Preserving the $N$-linear form (9) in dealing with the mutually correlated systems $\mathcal{M}^{\prime \ldots[i] \ldots t}$ is due to the above assumption of the relative weakness of the correlation mechanisms when the partial weights $\Omega^{\prime \ldots[i] \ldots l}$ of the systems $\mathcal{M}^{\prime \ldots[i] \ldots,} i=1, \ldots, N$, are not affected by the correlations.

We now intend to prove that the solution to the functional Eq. (8) for arbitrary values of the parameter $\omega$ coincides with the Tsallis entropy (5) provided that relation (9) can be applied. Assume, for simplicity, that the equiprobable distribution for the complex system $\mathcal{M}=\bigcup_{i=1}^{N} \mathcal{M}^{\prime \ldots[i] \ldots \prime}$ is the case. Substituting $S(\Omega) \equiv$ $[\sigma(\Omega)-1] / \omega$ into Eq. (8) and considering Eq. (9), after simple algebra one obtains

$\sigma\left(\prod_{i=1}^{N} \Omega^{\prime \ldots[i] \ldots t}\right)=\prod_{i=1}^{N} \sigma^{\prime \ldots[i] \ldots \prime}\left(\Omega^{\prime \ldots[i] \ldots \prime}\right)$.

A general functional solution to this equation is a power law $\sigma(\Omega)=\Omega^{\beta}$ where $\beta$ is a constant. Consequently, the entropy $S(\Omega)$ becomes

$S(\Omega)=\frac{\Omega^{\beta}-1}{\omega}$.

One then notices that the basic functional Eq. (8) doesn't contain any other auxiliary parameters besides $\omega$. Hence, any parameter $\beta$ which might appear in a solution to $\mathrm{Eq}$. (8), must be a function of $\omega$, i.e., $\beta=\beta(\omega)$. We now require that the right hand side of $\mathrm{Eq}$. (11) tends to the Boltzmann form, $\ln \Omega$, for $\omega \rightarrow 0$. [Note that in this case Eq. (8) crosses over to the functional equation of the form of Eq. (6), i.e,; $S(\Omega)=\sum_{i=1}^{N} S^{\prime \ldots[i] \ldots \prime}\left(\Omega^{\prime \ldots[i] \ldots \prime}\right)$, which doesn't contain any multiplicative terms and whose solution is the Boltzmann entropy, $S^{B}=\ln \Omega$.] This immediately yields $\beta(\omega)=\omega+\mathcal{O}\left(\omega^{2}\right)$ where $\mathcal{O}\left(\omega^{2}\right)$ denotes the feasible nonlinear terms in the Taylor expansion of $\beta(\omega)$ at $\omega \rightarrow 0$. We neglect these terms in what follows. Setting $\beta(\omega)=\omega$, from Eq. (11) one gets

$S(\Omega)=\frac{\Omega^{\omega}-1}{\omega}$.

Expression (12) is easily seen to reproduce the Tsallis entropy $S_{q}^{T}$ [see Eq. (5)] with

$q=1-\omega$.

From Eq. (13) it is evident that $q \rightarrow 1$ for $\omega \rightarrow 0$; in this limit, $S_{q}^{T}$ recovers the Boltzmann notion of entropy, $S^{B}=\ln \Omega$. The "unconventional" cases when the parameter $q$ deviates from unity, i.e., $q \neq 1$, could be assigned, in view of Eqs. (8) and (13), to physical systems whose entropies are nonadditive functions of the entropies of the constituent subsystems $(\omega \neq 0)$ due to the intrinsic long-range correlations therein.

The role of the long-range correlations in a physical system could be recognized in an appearance of structures with the features of ordering. Such structures might be supported by the long-range correlation mechanisms operating in the system, and their description in the framework of the conventional statistical mechanics assuming the Boltzmann definition of entropy (2) might be inadequate. An occurrence of such structures might be termed "ordering through correlations" [cf. Nicolis and Prigogine (1977)]. We might speculate that the "ordering through correlations" could give rise, in particular, to the "coarse-grained" topology of the system when "grains" of various sizes are organized in correlated patterns. In this context, the statistical weight $\Omega$ in Eq. (12) could be interpreted as the number of "grains".

An evidence for the "ordering through correlations" in a physical system described by the Tsallis entropy (5),(12) might be recognized from the functional Eqs. (7),(8). Indeed, consider, for simplicity, a complex system $\mathcal{M}=\mathcal{M}^{\prime} \cup \mathcal{M}^{\prime \prime}$ composed of the two $(N=2)$ mutually correlated subsystems $\mathcal{M}^{\prime}$ and $\mathcal{M}^{\prime \prime}$ having the entropies $S^{\prime}$ and $S^{\prime \prime}$, respectively. Then the entropy $S$ of the entire system $\mathcal{M}$ is given by Eq. (7). [Note that Eq. (8) is reduced to Eq. (7) for $N=2$.] Assume, first, that the parameter $\omega$ in Eq. (7) is negative, i.e., $\omega \equiv-|\omega|, \omega \neq 0$. Negative $\omega$ would mean that the entropy $S$ of the system $\mathcal{M}$ is smaller than the algebraic sum of the entropies of the constituent subsystems, i.e., $S<S^{\prime}+S^{\prime \prime}$, with the entropy deficit of $|\omega| S^{\prime} S^{\prime \prime}$. It is widely known that the physical states with the lower entropy $S$ would have a higher degree of ordering; hence. the degree of ordering of the whole system $\mathcal{M}$ composed of the two mutually correlated subsystems $\mathcal{M}^{\prime}$ and $\mathcal{M}^{\prime \prime}$ would be higher (for $\omega<0$ ) than the degree of ordering of the physical state when $\mathcal{M}^{\prime}$ and $\mathcal{M}^{\prime \prime}$ are isolated of each other. Consequently, the formation of the system 
$\mathcal{M}$ from the isolated systems $\mathcal{M}^{\prime}$ and $\mathcal{M}^{\prime \prime}$ would be followed by a decrease of the total entropy $S$ (for a value of $\left.|\omega| S^{\prime} S^{\prime \prime}\right)$, and, therefore, by an occurrence of ordering in $\mathcal{M}=\mathcal{M}^{\prime} \cup \mathcal{M}^{\prime \prime}$ as an imprint of the correlations between $\mathcal{M}^{\prime}$ and $\mathcal{M}^{\prime \prime}$. In the opposite case of positive $\omega$, i.e., $\omega \equiv|\omega|, \omega \neq 0$, the degree of ordering of the system $\mathcal{M}=\mathcal{M}^{\prime} \cup \mathcal{M}^{\prime \prime}$ is less than the degree of ordering of the two isolated systems $\mathcal{M}^{\prime}$ and $\mathcal{M}^{\prime \prime}$; hence, the formation of the whole system $\mathcal{M}=\mathcal{M}^{\prime} \cup \mathcal{M}^{\prime \prime}$ would be followed by an increase of the total entropy $S$ (for $|\omega| S^{\prime} S^{\prime \prime}$ ) and by the corresponding violation (destruction) of the original ordering in the isolated $\mathcal{M}^{\prime}$ and $\mathcal{M}^{\prime \prime}$ (i.e., "disordering through correlations"). This suggests an interpretation of the parameter $\omega$ in Eq. (7) as the bifurcation parameter [Nicolis and Prigogine (1977)] which distinguishes the two generalized statistics possible (i.e., the one for $\omega<0$, and the one for $\omega>0$ ), describing the opposite types of behavior from the viewpoint of the ordering in the complex system $\mathcal{M}=\mathcal{M}^{\prime} \cup \mathcal{M}^{\prime \prime}$. The zero value of the bifurcation parameter $\omega$, i.e., $\omega=0$, would recover the conventional statistical mechanics based on the Boltzmann definition of entropy (2), for which the above "ordering (disordering) through correlations" is absent.

\section{Establishing of the Canonical Distribution for the Tsallis Entropy}

Establishing of the canonical distribution for the Tsallis entropy $(5),(12)$ is straightforward and follows general methods of statistical mechanics [Landau and Lifshitz (1970)]. Let us divide the entire macroscopic system $\mathcal{M}$ into two parts, a small subsystem $\mathcal{M}_{\varepsilon}^{\prime}$ (which is treated below as a small macroscopic part of $\mathcal{M}$ ), and the residual (macroscopic) part $\mathcal{M}^{\prime \prime}$, so that $\mathcal{M}=\mathcal{M}_{\varepsilon}^{\prime} \cup \mathcal{M}^{\prime \prime}$. (The subscript " $\varepsilon$ " in the notation of $\mathcal{M}_{\varepsilon}^{\prime}$ is introduced to underline the fact that $\mathcal{M}_{\varepsilon}^{\prime}$ is only a very small part of $\mathcal{M}$. The system $\mathcal{M}$ is assumed to be in an equilibrium.) From the viewpoint of statistical mechanics, $\mathcal{M}$ can be considered as a "closed" system, and $\mathcal{M}^{\prime \prime}$, as the "heat bath" for $\mathcal{M}_{\varepsilon}^{\prime}$. Our goal would be to obtain the probability, $w$, of such a state of the entire system $\mathcal{M}$ when the subsystem $\mathcal{M}_{\varepsilon}^{\prime}$ is found in a microscopic state with given energy $E_{\varepsilon}^{\prime}$. (In the meanwhile, the corresponding microscopic state of the heat bath, $\mathcal{M}^{\prime \prime}$, is assumed to be arbitrary.)

Suppose $\mathcal{M}^{\prime \prime}$ is found in a microscopic state with the energy $E^{\prime \prime}$; one immediately concludes that the sum $E_{\varepsilon}^{\prime}+E^{\prime \prime}$ is equal to the energy, $E$, of the entire closed system $\mathcal{M}$, i.e., $E=E_{\varepsilon}^{\prime}+E^{\prime \prime}$. Then, denote $d \Omega^{\prime \prime}$ to be the statistical weight of all the microscopic states of the heat bath $\mathcal{M}^{\prime \prime}$ with the energies lying between $E^{\prime \prime}$ and $E^{\prime \prime}+d E^{\prime \prime}$; it is clear that $d \Omega^{\prime \prime}$ is a function of only $E^{\prime \prime}$, i.e., $d \Omega^{\prime \prime} \equiv d \Omega^{\prime \prime}\left(E^{\prime \prime}\right)$. This makes it possible to represent the desired probability $w$ as an integral in the
$\Omega^{\prime \prime}$-space [Landau and Lifshitz (1970)]:

$w=$ const $\times \int \delta\left(E_{\varepsilon}^{\prime}+E^{\prime \prime}-E\right) d \Omega^{\prime \prime}\left(E^{\prime \prime}\right)$

where $\delta$ is the Dirac delta-function. [Eq. (14) defines the microcanonical distribution for the heat bath $\mathcal{M}^{\prime \prime}$.] Because the expression under the integral sign in Eq. (14) depends only on $E^{\prime \prime}$, we can replace the integration over $\Omega^{\prime \prime}$ in Eq. (14) for the integration over $E^{\prime \prime}$ by using the obvious identity

$d \Omega^{\prime \prime}=\frac{d \Omega^{\prime \prime}\left(E^{\prime \prime}\right)}{d E^{\prime \prime}} d E^{\prime \prime}$

Next, the derivative $d \Omega^{\prime \prime}\left(E^{\prime \prime}\right) / d E^{\prime \prime}$ can be approximated by the ratio $\Delta \Omega^{\prime \prime} / \Delta E^{\prime \prime}$; here, $\Delta \Omega^{\prime \prime}=\int d \Omega^{\prime \prime}$ is the statistical weight of the macroscopic state of $\mathcal{M}^{\prime \prime}$ and is obtained by integrating the weights of all the microscopic states involved, whereas $\Delta E^{\prime \prime}$ is the energy range for $\mathcal{M}^{\prime \prime}$ which corresponds to the weight $\Delta \Omega^{\prime \prime}$. The quantity $\Delta \Omega^{\prime \prime}$ can be related to the entropy $S^{\prime \prime}\left(\Delta \Omega^{\prime \prime}\right)=$ $\left[\Delta \Omega^{\prime \prime} \omega-1\right] / \omega$, according to Eqs. (5) and (12); this yields the weight $\Delta \Omega^{\prime \prime}$ as the function of $S^{\prime \prime}$ by means of the simple expression $\Delta \Omega^{\prime \prime}=\left[1+\omega S^{\prime \prime}\right]^{1 / \omega}$. Considering $S^{\prime \prime}$ as a function of the energy $E^{\prime \prime}$, from Eq. (15) one gets

$\frac{d \Omega^{\prime \prime}}{d E^{\prime \prime}}=\frac{\left[1+\omega S^{\prime \prime}\left(E^{\prime \prime}\right)\right]^{1 / \omega}}{\Delta E^{\prime \prime}}$.

Consequently, Eq. (14) becomes

$$
\begin{aligned}
& w=\text { const } \times \\
& \quad \times \int\left[1+\omega S^{\prime \prime}\left(E^{\prime \prime}\right)\right]^{1 / \omega} \delta\left(E_{\varepsilon}^{\prime}+E^{\prime \prime}-E\right) \frac{d E^{\prime \prime}}{\Delta E^{\prime \prime}} .
\end{aligned}
$$

In view of the delta-function, the integration in Eq. (17) is reduced to the value of the integrand at $E^{\prime \prime}=E-E_{\varepsilon}^{\prime}$ : yielding

$$
w=\text { const } \times\left(\frac{\left[1+\omega S^{\prime \prime}\left(E^{\prime \prime}\right)\right]^{1 / \omega}}{\Delta E^{\prime \prime}}\right)_{E^{\prime \prime}=E-E_{\varepsilon}^{\prime}}
$$

Note, further, that the energy $E_{\varepsilon}^{\prime}$ of the system $\mathcal{M}_{\varepsilon}^{\prime}$ is negligible compared with $E$; this is because $\mathcal{M}_{\varepsilon}^{\prime}$ was assumed to be only a very small macroscopic part of the entire system $\mathcal{M}$. Relative changes in $\Delta E^{\prime \prime}$ caused by the variations of $E^{\prime \prime}$ of the order of $E_{\varepsilon}^{\prime}$ are also negligible. Hence, in Eq. (18), we can set $\Delta E^{\prime \prime} \approx \Delta E^{\prime \prime}(E)=$ const, neglecting the terms proportional to $E_{\varepsilon}^{\prime}$. Meanwhile, in the exponential term $\left[1+\omega S^{\prime \prime}\left(E^{\prime \prime}\right)\right]^{1 / \omega}$, we are expanding $S^{\prime \prime}\left(E-E_{\varepsilon}^{\prime}\right)$ in the Taylor series to give

$S^{\prime \prime}\left(E-E_{\varepsilon}^{\prime}\right)=S^{\prime \prime}(E)-E_{\varepsilon}^{\prime} \frac{d S^{\prime \prime}(E)}{d E}$

where the term proportional to $E_{\varepsilon}^{\prime}$ must be preserved. The derivative $d S^{\prime \prime}(E) / d E$ could be now defined as the 
inverse "thermodynamic temperature", $T$, of the system $\mathcal{M}$, i.e.,

$$
\frac{d S^{\prime \prime}(E)}{d E} \equiv \frac{1}{T}
$$

Definition (20) formally coincides with the standard definition [Landau and Lifshitz (1970)] of the thermodynamic temperature $T$ in an equilibrium, which assumes the Boltzmann form (2) for the entropy, $S^{\prime \prime}$, of the heat bath $\mathcal{M}^{\prime \prime}$. In the context of our study, however, we imply the generalized, Tsallis form (5),(12) for the entropy $S^{\prime \prime}$, rather than the Boltzmann form (2). This generalized form stands for the role of the long-range correlation mechanisms operating in the system $\mathcal{M}$. Hence, an extended sense must me assigned to definition (20): Eq. (20) defines the inverse thermodynamic temperature of the system $\mathcal{M}$ in an equilibrium when the long-range correlation effects are significant and affect the energy distribution throughout $\mathcal{M}$ [see Eq. (7)]. We also note that both the subsystem $\mathcal{M}_{\varepsilon}^{\prime}$ and the heat bath $\mathcal{M}^{\prime \prime}$ have the same generalized thermodynamic temperature $T$ in the equilibrium.

Combining Eqs. (19) and (20), from Eq. (18) we have

$w=$ const $\times\left(1-\frac{\omega}{1+\omega S^{\prime \prime}(E)} \frac{E_{\varepsilon}^{\prime}}{T}\right)^{1 / \omega}$,

yielding the probability distribution for the system $\mathcal{M}_{\varepsilon}^{\prime}$ which is in an equilibrium with the heat bath $\mathcal{M}^{\prime \prime}$. This distribution, however, should depend only very slightly on the entropy $S^{\prime \prime}$ of the heat bath $\mathcal{M}^{\prime \prime}$. [If this is not the case, one comes to an explicit nonlinear relationship between the probabilities of the microscopic states of the system $\mathcal{M}_{\varepsilon}^{\prime}$, and the allowed microscopic realizations for the heat bath $\mathcal{M}^{\prime \prime}$ which are described by the entropy $S^{\prime \prime}$. This, in turn, would imply that the microscopic configurations for $\mathcal{M}_{\varepsilon}^{\prime}$ and $\mathcal{M}^{\prime \prime}$ are correlated to a relatively high extent associated with a substantial reorganization of the microscopic states throughout $\mathcal{M}=\mathcal{M}_{\varepsilon}^{\prime} \cup \mathcal{M}^{\prime \prime}$. Such a conclusion is in contradiction with the above assumption of weakness of the correlation effects (see section 2); this assumption has been quantified by the functional Eqs. (7),(8) which are linear over each of the partial entropies involved and, therefore, neglect the possible nonlinear modifications of the corresponding microscopic states.]

Consequently, the probability distribution (21) is consistent with the functional form of Eqs. (7),(8) only if the following condition holds

$|\omega| S^{\prime \prime}(E) \ll 1$.

Hence, Eq. (21) could be finally written as

$w=A\left(1-\omega \frac{E_{\varepsilon}^{\prime}}{T}\right)^{1 / \omega}$

where the normalizing constant $A$ is defined from the obvious condition $T^{-1} \int w d E_{\varepsilon}^{\prime}=1$ to give $A=1+\omega$.
Expression (23) yields the probability distribution for a macroscopic system $\mathcal{M}_{\varepsilon}^{\prime}$ in an equilibrium provided that $\mathcal{M}_{\varepsilon}^{\prime}$ is a small part of a large closed system $\mathcal{M}$. In other words, Eq. (23) defines the canonical distribution for the system $\mathcal{M}_{\varepsilon}^{\prime}$ obeying the Tsallis definition (5),(12) of the concept of entropy. In the limiting case $\omega \rightarrow 0$ [which recovers the standard, Boltzmann notion of entropy (2)], the distribution (23) crosses over to the standard canonical (i.e., Gibbs) distribution, $w=\exp \left(-E_{\varepsilon}^{\prime} / T\right)$, which is widely known in statistical mechanics. The deviation of expression (23) from the purely exponential form $w=\exp \left(-E_{\varepsilon}^{\prime} / T\right)$ is due to the long-range correlations in the system $\mathcal{M}$ that affect the probabilities of the corresponding microscopic states.

Considering Eq. (12), one concludes that inequality (22) is equivalent to

$|\omega| \ln \Delta \Omega^{\prime \prime} \ll 1$,

where $\Delta \Omega^{\prime \prime}=\int d \Omega^{\prime \prime}$ is the statistical weight of the macroscopic state of the heat bath $\mathcal{M}^{\prime \prime}$ [see Eq. (15)]. Under the logarithm sign, we can also replace the weight $\Delta \Omega^{\prime \prime}$ for $\Delta \Omega$, this being the statistical weight of the macroscopic state of the entire system $\mathcal{M}$ corresponding to the energy $E$. Inequality (24) can be then rewritten in the suitable form

$|\omega| \ll \ln ^{-1} \Delta \Omega$.

This inequality shows that the parameter $\omega$ goes to zero for the system $\mathcal{M}$ whose statistical weight $\Delta \Omega$ is sufficiently large, i.e., $\Delta \Omega \rightarrow \infty$. For such $\mathcal{M}$, the effect of the long-range correlations satisfying the Tsallis notion of entropy (5),(12), can be fairly neglected, and the conventional statistical mechanics [Landau and Lifshitz (1970)] based on the Boltzmann definition of entropy (2) could be applied. One might assume, for instance, that the long-range correlation mechanisms in the system $\mathcal{M}$ could be practically neglected for the values of $|\omega|$ less than some $|\omega|_{0} \ll 1$, where $|\omega|_{0}$ is a small parameter describing the relative role of all the mechanisms counteracting the long-range correlation effects (e.g., thermal fluctuations, etc). Then the applicability of the Boltzmann entropy (2) would require the condition $\Delta \Omega \gg \Delta \Omega_{\min } \sim \exp \left(1 /|\omega|_{0}\right)$, instead of the less certain $\Delta \Omega \rightarrow \infty$. In the meanwhile, for the systems $\mathcal{M}$ with relatively small statistical weights $\Delta \Omega \leq \Delta \Omega_{\min } \sim \exp \left(1 /|\omega|_{0}\right)$, the Tsallis definition of entropy (5),(12) might have physical sense, leading to the "unconventional" canonical distribution (23). Consequently, the generalized statistical mechanics based on the Tsallis entropy (5),(12) must imply the limitation $\Delta \Omega \leq \exp \left(1 /|\omega|_{0}\right)$ on the statistical weight of the system $\overline{\mathcal{M}}$, providing the dominant role of the long-range correlations in $\mathcal{M}$.

The condition $\Delta \Omega \leq \exp \left(1 /|\omega|_{0}\right)$ might be naturally satisfied for the "coarse-grained" systems discussed in section 2 in the context of the "ordering through correlations". General thermodynamic properties of the 
"coarse-grained" systems are analyzed in detail in, e.g., the monographs of Gibbs (1960), Toleman (1979), and Gallavotti (1999). The idea of the "coarse-graining" has been applied by Tetreault (1992ab) to a study of selforganization and intermittency in the stochastic magnetic fields. Similar issues were exploited by Milovanov et al. $(1996,2000)$ and Zelenyi et al. (1998) in relationship with the self-organized percolation structuring in the distant Earth's magnetotail.

The canonical distribution (23) coincides, for negative $\omega$, with the so-called "kappa" distribution which plays an important role in many applications. It is remarkable to note that just negative values of $\omega$ can be associated with the "ordering through correlations" and the formation of the "coarse-grained" correlated patterns. This might be a hint to consider the "kappa" distributions (23) as a direct manifestation of the "macroscopic" ordering in the system and the ensueing development of the "coarse-grained" structures. We shall come back to this issue in the end of section 4.

The motivation for using "kappa" distribution functions in literature mostly follows interpretational purposes and still lacks proper theoretical basis:

Christon et al. (1989) reported a "kappa" distribution from a comprehensive study of the plasma particle populations in the near-Earth environment. A "kappa" distribution was indicated by Winningham (1992) for the accelerated particles in the Earth's magnetotail. The "kappa" distributions of electrons and protons accelerated in the solar corona were assumed by Maksimovic et al. (1997) in the context of the kinetic model of the solar wind.

Except for Hasegawa et al. (1985) who substantiated the "kappa" distribution of a plasma immersed in a superthermal radiation field, and Collier (1993) who could generate a "kappa"-like distribution function from random walk jumps in velocity space, only few authors discussed the physical origin of the "kappa" distributions.

Ma and Summers (1998) found that a "kappa" distribution could be the result of the stochastic particle acceleration by whistler-mode turbulence; Summers and Thorne (1992) also recognized that a "kappa" distribution can itself enhance the whistler-mode instability.

Zelenyi and Milovanov (1992) demonstrated that the "kappa" distributions provide an extended Lie group symmetry for the Vlasov-Maxwell equations; moreover, having solved the Lie group classification problem, they could rigorously prove that the "kappa" distributions are the only functions (along with the standard Gibbs distribution corresponding to the limit $\omega \rightarrow 0$ ) for which the extended symmetries could be principally allowed.

An important property of the "kappa" distribution (23) is the evidence for the power-law "tail" for $\omega<0$. Indeed, setting $\omega \equiv-|\omega|$ and $E_{\varepsilon}^{\prime} \gg T /|\omega|$, from Eq. (23) one gets

$w \propto E_{\varepsilon}^{\prime-\gamma}$ where $\gamma=1 /|\omega|$. [For the small values of $E_{\varepsilon}^{\prime}$, i.e., $E_{\varepsilon}^{\prime} \ll T /|\omega|$, Eq. (23) yields the standard, exponential distribution $w \propto \exp \left(-E_{\varepsilon}^{\prime} / T\right)$ independently of the sign of $\omega$.] Rigorously speaking, the power exponent $\gamma$ in Eq. (26) must be sufficiently large, in view of condition (25), so that distribution (26) decreases relatively fast with the energy $E_{\varepsilon}^{\prime}$.

The power-law distributions of the form (26) are commonly assigned to physical systems involving coherent mechanisms (e.g., coherent acceleration mechanisms for charged particles). An example might be the statistics of cosmic rays [Berezinsky et al. (1990)] revealing the power-law energy spectrum (26) over a logarithmically wide range of energies.

An impact of the coherent mechanisms could be found in the energy transfer towards higher (for $\omega<0$ ) energy range of the spectrum, leading to the formation of the power-law "tail" (26). In this context, the power exponent $\gamma=1 /|\omega|, \omega<0$, in distribution (26) might be interpreted as a "degree of coherence" of the basic mechanisms involved. For instance, a more "flat" spectrum (26), i.e., the one having a smaller value of the power exponent $\gamma$, would correspond to a higher degree of coherence of the basic mechanisms effective in the system; this appears in a larger value of $|\omega|$ and in a more pronounced power-law "tail" of the canonical distribution (23).

The total energy, $\delta E$, transferred towards the "tail" (26), could be roughly estimated by integrating the deviation, $\Delta w$, of distribution (23) from its purely exponential form, $w=\exp \left(-E_{\varepsilon}^{\prime} / T\right)$, at $\omega=0$. Expanding $\Delta w$ in the Taylor series over the small parameter $\omega$ and keeping, for simplicity, only the first (i.e., linear) term, one finds, approximately,

$\delta E \approx \int_{0}^{\infty}\left\{\left.\omega \frac{d w}{d \omega}\right|_{\omega=0}\right\} d E_{\varepsilon}^{\prime}$.

Simple calculations lead to the result

$\delta E \approx-2 \omega T$

where Eq. (23) has been used. Eq. (28) shows that the energy $\delta E$ is positive for $\omega<0$, and negative, for $\omega>0$.

The energy $\delta E$ might be effectively gained by a small fraction, $\delta \mathcal{N} / \mathcal{N} \ll 1$, of the "resonance" particles, whose presence is required by some acceleration mechanisms [see, e.g., Berezinsky et al. (1990)]. [Here, $\mathcal{N}$ is the particle number density. We also note that the energy $\delta E$ in Eq. $(28)$ is normalized to one particle $(\mathcal{N}=1)$ as it follows from the normalizing condition $T^{-1} \int w d E_{\varepsilon}^{\prime}=1$ assumed in the canonical distribution (23). For arbitrary values of $\mathcal{N}$, Eq. (28) should be replaced for $\delta E \approx-2 \mathcal{N} \omega T$.] Hence, the "resonance" particles can be accelerated to the typical energies

$\mathcal{E} \sim 2|\omega| T \frac{\mathcal{N}}{\delta \mathcal{N}}, \quad \omega<0$. 
These energies may exceed the characteristic thermal energy, $T$, for the relatively large values of the parameter $|\omega| \geq \delta \mathcal{N} / 2 \mathcal{N}$.

It is worth mentioning that a treatment of physical systems exhibiting coherent mechanisms might require an inclusion of rather artificial auxiliary constraints if based on the statistical mechanical formalism assuming the Boltzmann definition of entropy (2) [see, also, the relevant discussion in Alemany and Zanette (1993)]. In the meanwhile, an alternative approach [which could be based on the generalized definition of entropy in the Tsallis form (5),(12)] enables one to preserve the conventional framework of calculus as shown above (this framework assumes the standard statistical mechanical constraints) in dealing with the systems with coherence, on only account of the suitable generalization of the concept of entropy. In particular, this follows from the fact that the generalized distribution (23) [which contains the power-law "tail" (26)] could be obtained as the canonical distribution for the Tsallis entropy (5),(12) within the conventional statistical mechanical analysis.

In conclusion, we note that the canonical distribution (23) implicitly assumes that the total populations of the microscopic states for each subsystem of the system $\mathcal{M}$ are time-invariant, i.e., no matter flows between the subsystems of $\mathcal{M}$ are allowed. An inclusion of such flows (which result in variations of the total populations of the subsystems) might be done through an introduction of the chemical potential $\Upsilon$ [Landau and Lifshitz $(1970)$ ]; in this case, $E_{\varepsilon}^{\prime}$ in Eq. (23) must be replaced for $E_{\varepsilon}^{\prime}-\Upsilon$. The distribution $w$ obtained in this way will be the grand canonical distribution for $\mathcal{M}_{\varepsilon}^{\prime}$ corresponding to the Tsallis entropy (5),(12).

\section{Summary and Conclusions}

Summarizing the results obtained, we mention that the generalized entropy (5) originally postulated by Tsallis (1988) in his pioneering paper, can be rigorously derived as the solution to the functional equation (8) under condition (9). This functional equation quantifies the behavior of the entropy, $S$, of the complex system $\mathcal{M}=\bigcup_{i=1}^{N} \mathcal{M}^{\prime \cdots[i] \ldots t}$ through the partial entropies, $S^{\prime \ldots[i] \ldots t}$, of the constituent subsystems $\mathcal{M}^{\prime \ldots[i] \ldots t}$ and assumes the presence of the long-range correlation mechanisms affecting the microscopic probability distributions for the subsystems involved.

Eqs. (8), (9) take into account the correlation effects up to the highest order, $N \geq 2$, effective in the complex system $\mathcal{M}=\bigcup_{i=1}^{N} \mathcal{M}^{\prime \ldots[i] \ldots \prime}$. For instance, the correlations of the order $2 \leq n \leq N$ are quantified by $C_{N}^{n}=N ! / n !(N-n) !$ multiplicative terms on the right of the functional Eq. (8); each of these terms has the form of an $n$-linear product, $\omega^{n-1} S^{\prime \ldots[i] \ldots /} S^{\prime \ldots[j] \ldots \prime} \times$ $\ldots \times S^{\prime \ldots[l] \ldots \prime}, i<j<\ldots<l$, of $n$ partial entropies $S^{\prime \ldots[i] \ldots t}, S^{\prime \ldots[j] \ldots \prime}, \ldots, S^{\prime \ldots[i] \ldots t}$ for $n$ different subsystems
$\mathcal{M}^{\prime \ldots[i] \ldots t}, \mathcal{M}^{\prime \ldots[j] \ldots t}, \ldots, \mathcal{M}^{\prime \ldots[l] \ldots t}$ of the system $\mathcal{M}$. The functional Eq. (8) is thus linear over each of the partial entropies $S^{\prime \cdots[i] \ldots t}, i=1, \cdots, N$, and contains in total $2^{N}-(N+1)$ multiplicative terms of all the orders $n$ from 2 to $N \geq 2$. The linearity of the basic Eq. (8) over each of the entropies $S^{\prime \ldots[i] \ldots \prime}$ implies that the correlation mechanisms are sufficiently weak to change the statistical weights, $\Omega^{\prime \ldots[i] \ldots \prime}$, of the subsystems $\mathcal{M}^{\prime \ldots[i] \ldots \prime}$, enabling one to preserve the conventional representation (9) of the statistical weight, $\Omega$, of the complex system $\mathcal{M}=\bigcup_{i=1}^{N} \mathcal{M}^{\prime \ldots[i] \ldots \prime}$ as $\prod_{i=1}^{N} \Omega^{\prime \ldots[i] \ldots \prime}$.

The basic parameter $\omega$ which appears on the right of the functional Eq. (8), describes the strength of the correlations; the correlations of the order $2 \leq n \leq N$ are then signified by the $(n-1)$-th power of $\omega$, i.e., $\omega^{n-1}$, this being the corresponding correlation amplitude for the multiplicative term involved, $\omega^{n-1} S^{\prime \ldots[i] \ldots t} S^{\prime \ldots[j] \ldots t} \times$ $\ldots \times S^{\prime \ldots[l] \ldots \prime}$. The weakness of the correlation mechanisms is clear from inequality (25) and leads to the exponential decay $\left(\propto \omega^{n-1}\right)$ of the correlation amplitudes versus $n$.

For $\omega \rightarrow 0$ (when the correlation effects are negligible), the standard, Boltzmann definition of entropy (2) is recovered from Eq. (8). The Boltzmann entropy (2) reveals the property of additivity; this property is contained in the linear functional form of the basic Eq. (8) for $\omega \rightarrow 0$ [see, e.g., Eq. (6)]. On the contrary, the property of additivity is violated for the generalized, Tsallis entropy (5),(12) assuming the nonzero values of the parameter $\omega$. The essential nonadditivity of the Tsallis entropy (5),(12) for the nonvanishing values of $\omega$ is explicitly present in the multiplicative functional terms on the right of Eq. (8) and stands for the role of the long-range correlation mechanisms.

We could speculate that the nonadditivity of the Tsallis entropy (5),(12) might be associated with an occurrence of structures with the features of ordering. This could be seen from the functional Eqs. (7),(8) which allow an entropy deficit for complex systems as a result of the inclusion of the long-range correlations (i.e., the "ordering through correlations"). The "ordering through correlations" might support the "coarse-grained" topology of the system when "grains" of various sizes are assembled in correlated patterns. The statistical weight $\Omega$ in the Tsallis entropy (12) should be then interpreted as the number of the "grains" involved.

Our results indicate that the role of the long-range correlations described by the functional Eqs. (7), (8) might be essential only for the systems with the relatively small statistical weights $\Omega$ not exceeding the value of $\sim \exp \left(1 /|\omega|_{0}\right)$; the $|\omega|_{0}$ is a small parameter which quantifies the corresponding role of the mechanisms counteracting the long-range correlation effects (e.g., thermal fluctuations, etc). Hence, an application of the generalized statistics based on the Tsallis definition of entropy (5),(12) might have sense only for 
the systems with the statistical weights less than $\sim$ $\exp \left(1 /|\omega|_{0}\right)$. In the opposite case of the systems with the weights much larger than $\sim \exp \left(1 /|\omega|_{0}\right)$, the conventional statistical mechanics must be used which is based on the Boltzmann entropy (2).

This remark has important consequences for the thermodynamics of the "coarse-grained" systems. Indeed, one concludes that the Tsallis entropy (5),(12) could be applied only at the "macroscopic" level associated with the "coarse-grained" structure of the system; in the meantime, at the "microscopic" scales (i.e., well "inside" the "grains"), the conventional Boltzmann statistics remains the case. Hence, the full entropy of the "coarsegrained" system should be treated as the sum of the "macroscopic" Tsallis entropy (5),(12) defined for the ensemble of the "grains", and the "microscopic", Boltzmann entropy (2) characterizing the disorder inside each "grain".

The consideration of the full entropy of the system resolves the concerns about the allowed "violation" of the second law in the Tsallis thermodynamics [see, e.g., Treumann (1999a), Treumann (1999b)]. Indeed, for the open systems, the Tsallis entropy deficit associated with the "macroscopically" ordered structures, could be naturally maintained due to the entropy outflows into the exterior medium [Nicolis and Prigogine (1977)]. These outflows could stabilize the system far from the thermal equilibrium, and the "coarse-grained" patterns could permanently exist as the effect of the correlations involved. Similar situation might be also realized in the closed thermodynamic systems far from equilibrium, but only for finite time intervals. In this case, an appearance of the "macroscopically" ordered structures like the "coarse-grained" correlated patterns might be the consequence of the violent relaxation at the "microscopic" scales. In other words, the inhibited growth of the Tsallis entropy leading to a (temporary) "coarsegraining" of the system is "compensated" by the entropy "overproduction" inside the "grains", so that the total entropy growth (which takes into account the relevant contributions from both the macro- and micro-scales) satisfies the $H$-theorem. Once the thermal equilibrium is achieved, no "macroscopically" ordered structures could, of course, occur any more in the closed system.

It rnight be worth to compare these conclusions with the recent findings of Treumann (1999ab). Treumann studied the evolution of the closed, nearly collisionless system with long-range correlations from the state with the sufficient amount of free energy towards the thermal equilibrium. He found that the system evolves through the strongly nonlinear stage (the so-called "turbulent quasi-equilibrium") characterized by the coexistence of many different mutually interconnected scales affecting each other. These turbulence structures could correspond, in our language, to the "coarse-grained" correlated patterns whose feasible development preceeds the thermal equilibrium state.

Then, Treumann introduces the correlations in the effective collisional term which contains a power law dependence as the suitable approximation of the multiscale interconnections in the system. This is an alternative of the inclusion of the correlations directly into the multiplicative terms yielding the expansion (8) of the entropy functional for the complex system with the interconnections.

Analysing the kinetic equation with the effective collisional term, Treumann could construct the "turbulent entropy" $\mathcal{S}$ (defined as the ensemble average over the logarithm of the correlation functional) which clearly satisfies the $H$-theorem and couldn't be reduced to only the standard Tsallis form (12). One might propose that the "turbulent entropy" $\mathcal{S}$ is the practical approximation of the full entropy of the closed system at "turbulent quasi-equilibrium", which combines the contributions from macro- (Tsallis) and micro- (Boltzmann) scales into the effective united form. It is also interesting to note that the "turbulent quasi-equilibrium" is characterized by relatively slow growth of the entropy $\mathcal{S}$; this might substantiate the assumed formation of the turbulence "coarse-grained" correlated patterns associated with an occurrence of the entropy deficit at the "macroscopic" scales.

The solution of the kinetic equation with the effective collisional term on the right hand side leads to the "kappa"-like distribution function at the "lurbulent quasi-equilibrium" [Treumann (1999a), Treumann (1999b)]. This "kinetic" result practically coincides with the canonical distribution (23) obtained in our study within purely thermodynamic formalism. This substantiates the principal equivalence of the Treumann's "kinetic" approach [Treumann (1999ab)] to the "thermodynamic" treatment of the "coarse-grained" closed systems far from equilibrium on the basis of the Tsallis entropy.

The comprehension of the "kappa"-like distribution functions as the canonical distributions (23) for the Tsallis entropy (5), (12) opens new perspectives on the "macroscopically" ordered (i.e., "coarse-grained") systems. Indeed, an appearance of the "kappa" distributions could be treated as the direct manifestation of the long-range correlations operating in the system and "deforming" the spectral energy density due to the "fragmentation" into the interconnected "grains".

The close relationship between the "macroscopic" ordering and formation of the "kappa" distributions conld be understood from the following general arguments. The "ordering" requires an entropy deficit which could be associated with negative heat $-\Delta Q$. The energy $\Delta Q$ released at the "macroscopic" scales as the result of the "ordering" would be accepted, in the closed system, by the "microscopic" particles inside the "grains". That is, the particles basically gain energy, and their spectral energy density increases at the higher energy range. For the multiscale interconnected "grains", no typical value 
of $\Delta Q$ could be defined above some characteristic level (of the order of the "thermal" energy, T.). Hence, the distribution of the particle energy density would have no particular scale at the higher energy range and is reduced, therefore, to the power-law form $w \propto E^{-\gamma}$. At the lower energy range, the "thermal" energy $T$ remains the only quantity describing the particle energy density distribution, and the "shape" of the particle distribution function could be defined as the (re-normalized) Gibbs distribution $w \propto \exp (-E / T)$. Thus, an evidence for the "kappa" distribution (i.e., the power law "tail" $\propto E^{-\gamma}$ at the higher energy range, and the Gibbs distribution $w \propto \exp (-E / T)$ at the lower energies $)$ indicates towards "macroscopic" ordering in the system, where the power exponent $\gamma$ characterizes the strength of the correlations involved.

In conclusion, it is important to emphasize that the concept of the Tsallis entropy (5),(12) enables one to deal with the "macroscopically" correlated systems in the framework of the conventional statistical mechanics (see section 3), whereas an exploitation of only the standard, Boltzmann entropy (2) would require an inclusion of rather artificial auxiliary conditions that often complicate the analysis.

Acknowledgements. This work was supported by the INTAS grant No. 97-1612 and Russian Foundation of Fundamental Research (projects No. 00-02-17127 and 00-15-96631).

\section{References}

Alemany, P.A., and Zanette, D.H., Fractal random walks from a variational formalism for Tsallis entropies, Phys. Rev. E, 49, R956-R958, 1993.

Badii, R., and Politi, A., Complexity. Hierarchical Structures and Scaling in Physics, Cambridge Univ. Press, Cambridge, 1997.

Berezinsky, V.S., Ginzburg, V.L., Bulanov, S.V., Dogiel, V.A., and Ptuskin, V.S., Astrophysics of Cosmic Rays, Nauka, Moscow, 1990, in Russian.

Boghosian, B.M., Thermodynamic description of the relaxation of two-dimensional turbulence using Tsallis statistics, Phys. Rev. $E, 53,4754-4763,1996$.

Buchner, J., Three-dimensional magnetic reconnection in astrophysical plasmas - Kinetic approach, Astrophys. Space Sci., 264, 25-42, 1998. [See, also, Buchner, J., in Physics of Space Plasmas 15, Eds. T. Chang, and J.R. Jasperse, (Massachusetts Institute of Technology, Cambridge, MA, 1998). p.383.]

Buyukkilic, F., and Demirhan, D., A fractal approach to entropy and distribution functions, Phys. Lett. A, 181, 24-28, 1993.

Chang, T., Self-organized criticality, multifractal spectra, sporadic localized reconnections and intermittent turbulence in the magnetotail, Phys. Plasmas, 6, 4137-4145, 1999.

Christon, S.P., Williams, D.J., Mitchell, D.G., Frank, L.A., and Huang, C.Y., Spectral characteristics of plasma sheet ion and electron populations during undisturbed geomagnetic conditions, J. Geophys. Res., 94, 13,409-13,424, 1989.

Collier, M.R., On generating kappa-like distribution functions using velocity space Levy flights, Geophys. Res. Lett., 20, 1531$1534,1993$.

Daroczy, Z., Inf. Control, 16, 36, 1970.

Eckmann, J.-P., and Ruelle, D., Ergodic theory of chaos and strange attractors, Rev. Mod. Phys., 57, 617-656, 1985.
Gaspard, P., and Dorfman, J.R., Chaotic scattering theory, thermodynamic formalism, and transport coefficients, Phys. Rev. $E, 52,3525-3552,1995$.

Gibbs, J.W., Elementary Principles in Statistical Mechanics, Yale Univ. Press, Yale, 1902; Dover, New York, 1960.

Gallavotti, G., Statistical Mechanics: A Short Treatise, Springer, Berlin, 1999.

Haldane, F.D.M., "Fractional statistics" in arbitrary dimensions: A generalization of the Pauli principle, Phys. Rev. Lett., 67, 937-940, 1991.

Hamity, V.H., and Barraco, D.E., Generalized nonextensive thermodynamics applied to the cosmic background radiation in a Robertson-Walker Universe, Phys. Rev. Lett., 76, 4664-4666, 1996.

Hasegawa, A., Mima, K., and Duong-van, M., Plasma distribution function in a superthermal radiation field, Phys. Rev. Lett., 54, 2608-2610, 1985.

Isichenko, M.B., Percolation, statistical topography, and transport in random media, Rev. Mod. Phys., 64, 961-1043, 1992.

Landau, L.D., and Lifshitz, E.M., Statistical Physics, Pergamon, Oxford, 1970.

Levich, V.G., Course of Theoretical Physics, Volume 1, Fizmatgiz, Moscow, 1962, in Russian.

Ma, C.-Y., and Summers, D., Formation of power-law energy spectra in space plasmas by stochastic acceleration due to whistlermode waves, Geophys. Res. Lett., 25, 4099-4102, 1998.

Maksimovic, M., Pierrard, V., and Lemaire, J.F., A kinetic model of the solar wind with Kappa distribution functions in the corona, Astron. Astrophys., 924, 725-734, 1997.

Mariz, A.M., On the irreversible nature of the Tsallis and Renyi entropies, Phys. Lett. A, 165, 409-411, 1992.

McCauley, J.L., Introduction to multifractals in dynamical system theory and fully developed fluid turbulence, Phys. Rep., 189, 225-266, 1990.

Milovanov, A.V., Topological proof for the Alexander-Orbach conjecture, Phys. Rev. E, 56, 2437-2446, 1997.

Milovanov, A.V., and Zelenyi, L.M., Fracton excitations as a driving mechanism for the self-organized dynamical structuring in the solar wind, Astrophys. Space Sci., 264, 317-345, 1998.

Milovanov, A.V., and Zimbardo, G., Percolation in signsymmetric random fields: Topological aspects and numerical modeling, Phys. Rev. E, 59, 2000 (in press).

Milovanov, A.V., Zelenyi, L.M., and Zimbardo, G., Fractal structures and power-law spectra in the distant Earth's magnetotail, J. Geophys. Res., 101, 19,903-19,910, 1996.

Milovanov, A.V., Zelenyi, L.M., Zimbardo, G., and Veltri, P., Selforganized branching of magnetotail current systems near the percolation threshold, J. Geophys. Res., 2000 (submitted).

Nakayama, T., Yakubo, K., and Orbach, R.L., Dynamical properties of fractal networks: Scaling, numerical simulations and physical realizations, Rev. Mod. Phys., 66, 381-443, 1994.

Nicolis, G., and Prigogine, I., Self-Organization in Nonequilibrium Systems. From Dissipative Structures to Order Through Fluctuations, Wiley, New York, 1977.

Paladin, G., and Vulpiani, A., Anomalous scaling laws in multifractal objects, Phys. Rep., 156, 147-225, 1987.

Plastino, A.R., and Plastino, A., Tsallis' entropy, Ehrenfest theorem and information theory, Phys. Lett. A, 177, 177-179, 1993.

Plastino, A., and Tsallis C., Variational method in generalized statistical mechanics, J. Phys. A, 26, L893-L896, 1993.

Rajagopal, A.K., Dynamic linear response theory for a nonextensive system based on the Tsallis prescription, Phys. Rev. Lett., $76,3469-3473,1996$.

Ramshaw, J.D., $H$-theorems for the Tsallis and Renyi entropies, Phys. Lett. A, 175, 169-170, 1993.

Ramshaw, J.D., Irreversibility and generalized entropies, Phys. Lett. A, 175, 171-172, 1993.

Reif, F., Fundamentals of Statistical and Thermal Physics, McGraw-Hill, New York, 1965. 
Renyi, A., On a new axiomatic theory of probability, Acta Mathematica Hungarica, 6, 285, 1955.

Renyi, A., Probability Theory, North-Holland, Amsterdam, 1970.

Ruelle, D., Thermodynamic Formalism, Addison-Wesley, Reading, Massachusetts, 1978.

Saslaw, W., Gravitational Physics of Stellar and Galactic Systems, Cambridge Univ. Press, Cambridge, 1987.

Schroeder, M., Fractals, Chaos, Power Laws, Freeman, New York, 1991.

da Silva E.P., Tsallis, C., and Curado, E.M.F., Specific heat of a free particle in a generalized Boltzmann-Gibbs statistics, Physica $A, 199,137-153,1993$.

Sinai, Ya.G., Introduction to Ergodic Theory, Princeton Univ. Press, Princeton, 1972.

Spitzer, L., Jr., Dynamical Evolution of Globular Clusters, Princeton Univ. Press, Princeton, 1987.

Summers, D., and Thorne, R.M., A new tool for analyzing microinstabilities in space plasmas modeled by a generalized Lorentzian (Kappa) distribution, J. Geophys. Res., 97, 16,827$16,832,1992$.

Tetreault, D., Turbulent relaxation of magnetic fields 1 . Coarsegrained dissipation and reconnection. J. Geophys. Res., 97, 8531-8540, 1992.

Tetreault, D., Turbulent relaxation of magnetic fields 2. Selforganization and intermittency, J. Geophys. Res., 97, 85418547,1992 .
Toleman, R.C., The Principles of Statistical Mechanics, Oxford Univ. Press, Oxford, 1938; Dover, New York, 1979.

Treumann, R.A., Kinetic theoretical foundation of Lorentzian statistical mechanics, Physica Scripta, 59, 19-26, 1999a.

Treumann, R.A., Generalized-Lorentzian thermodynamics, Physica Scripta, 59, 204-214, 1999b.

Tsallis, C., Possible generalization of Boltzmann-Gibbs statistics, J. Stat. Phys., 52, 479-487, 1988.

Winningham, J.D., preprint, 1992.

Witten, E., Gauge theories, vertex models, and quantum groups, preprint Princeton Advanced Study Inst., IASSNS-HEP-89/32, 1989; Gauge theories and integrable lattice models, preprint Princeton Advanced Study Inst., IASSNS-HEP-89/11, 1989. [See, also, Comm. Math. Phys., 121, 351, 1989].

Zanette, D.H., and Alemany, P.A., Thermodynamics of anomalous diffusion, Phys. Rev. Lett., 75, 366-369, 1995.

Zelenyi, L.M., and Milovanov, A.V., Applications of Lie groups to the equilibrium theory of cylindrically symmetric magnetic flux tubes, Sov. J. Astron. Zh., 69, 147-158, 1992. [Engl. Transl.: Sov. Astron., 36(1), 74-80, 1992.]

Zelenyi, L.M., Milovanov, A.V., and Zimbardo, G., Multiscale magnetic structure of the distant tail: Self-consistent fractal approach, in New Perspectives of the Earth's Magnetotail, Geophys. Monograph 105, Eds. A. Nishida, D.N. Baker, and S.W.H. Cowley, (American Geophysical Union, Washington, DC, 1998). p.321-339. 